\title{
Methods of Power Line Interference Elimination in EMG Signals
}

\author{
Martina Ladrova ${ }^{1, a}$, Radek Martinek ${ }^{1, b}$, Jan Nedoma ${ }^{2, c^{*}}$, Marcel Fajkus ${ }^{2, d}$ \\ ${ }^{1}$ Department of Cybernetics and Biomedical Engineering, Faculty of Electrical Engineering and \\ Computer Science, VSB-Technical University of Ostrava, Ostrava, Czech Republic \\ ${ }^{2}$ Department of Telecommunications, Faculty of Electrical Engineering and Computer Science \\ VSB-Technical University of Ostrava, Ostrava, Czech Republic
}

amartina.ladrova@vsb.cz, bradek.martinek@vsb.cz,c”jan.nedoma@vsb.cz, dmarcel.fajkus@vsb.cz

\begin{abstract}
Keywords: Electromyography, Biological signal processing, Power Line, Notch filter, Adaptive Noise Canceller, Wavelet Transform.
\end{abstract}

\begin{abstract}
Electromyogram (EMG) recordings are often corrupted by the wide range of artifacts, which one of them is power line interference (PLI). The study focuses on some of the well-known signal processing approaches used to eliminate or attenuate PLI from EMG signal. The results are compared using signal-to-noise ratio (SNR), correlation coefficients and Bland-Altman analysis for each tested method: notch filter, adaptive noise canceller (ANC) and wavelet transform (WT). Thus, the power of the remaining noise and shape of the output signal are analysed. The results show that the ANC method gives the best output SNR and lowest shape distortion compared to the other methods.
\end{abstract}

\section{Introduction}

Electromyogram (EMG) is a type of diagnostic method, which allows measuring of the biological signal that originates due to the skeletal muscle activity. The technique often consists of the stimulation of the relevant motoric and peripheral nerves and simultaneous measuring of the EMG signal. The measuring can be either invasive or non-invasive, monitoring the activity of single muscle fiber, one motor unit or whole muscle. The signal gives us the information which can be used for the diagnose of muscular and neuromuscular disease, walk analysis, rehabilitation or control the limb prosthesis.

The EMG signal seems to be very complicated in its analysis, because of its non-stationarity, which complicates the use of the filtration in a frequency domain, and wide frequency range from $10 \mathrm{~Hz}$ up to $1 \mathrm{kHz}$. The EMG signal, such as every biological signal, is influenced by many types of interference and artifacts. The power line interference (PLI) on frequency $50 \mathrm{~Hz}$ (in Europe) or $60 \mathrm{~Hz}$ (in North America), which is object of this paper, brings the more significant problem in EMG signal than in the case of the other biological signals due to the signal frequency range - the monitored range of other biological signals (e.g. ECG, EEG) could be reduced under $50 \mathrm{~Hz}$, which enables the use of the low pass filter. In the case of EMG, it is not possible to use the low pass filter because of its frequency up to $1 \mathrm{kHz}$ and it is necessary to use the notch filter or some of the other types of filtration, e.g. wavelet transform (WT) or adaptive filtering $[1,2,3]$.

The paper compares the results of the well-known methods of EMG signal filtration (notch filter, adaptive noise canceller (ANC) and WT) between the original artificially generated EMG signal and the filtered signal after mixing with the $50 \mathrm{~Hz}$ PLI. The MATLAB software was used for implementation of the filtration methods, and the correlation and signal-to-noise ratio (SNR) were computed for the effectiveness evaluation of each method.

\section{Material and Methods}

The software for biomedical signal processing was implemented in MATLAB. It represents the tool for testing and comparing the well-known processing and filtration methods used in onedimensional biological signal processing. The software allows loading signals from .mat file, adding the basic types of interference (high and low-frequency interference, power line interference, white 
noise or trends) to the signal and signal processing including FIR and IIR filtering, ANC, derivation, WT, independent and principal component analysis, etc. After each change of the processed signal (e.g. adding interference or filtering), the SNR and correlation coefficients are calculated to compare the effectiveness of each signal processing method. The signals are evaluated also by the graphical way - time waveforms, frequency spectra and spectrograms are shown for each type of signal original, corrupted and filtered (Fig. 1).

The paper targets on the EMG signal processing. The three methods of PLI removal are discussed in detail in the following subsections.

\subsection{Notch filter}

The notch filter is the simplest method for removing the fixed narrow bandwidth interference from the signal. The mathematical expression of its transfer function is defined in (1).

$$
H(z)=b_{0} \frac{1-2 \cos \omega_{0} z^{-1}+z^{-2}}{1-2 r \cos \omega_{0} z^{-1}+r^{2} z^{-2}}
$$

where $\omega_{0}$ is the central frequency of the interference and the width of the notch at $-3 \mathrm{~dB}$ is $2(1-\mathrm{r})$ $\mathrm{rad} / \mathrm{s}$.

Because the PLI frequency and its harmonics are located within the bandwidth of EMG, notch filter removes PLI as well as the components of EMG signal located at this frequency and few frequencies around. Therefore, the filter causes the information loss. For the distortion minimization, narrow band of the filter should be used $[1,3]$.

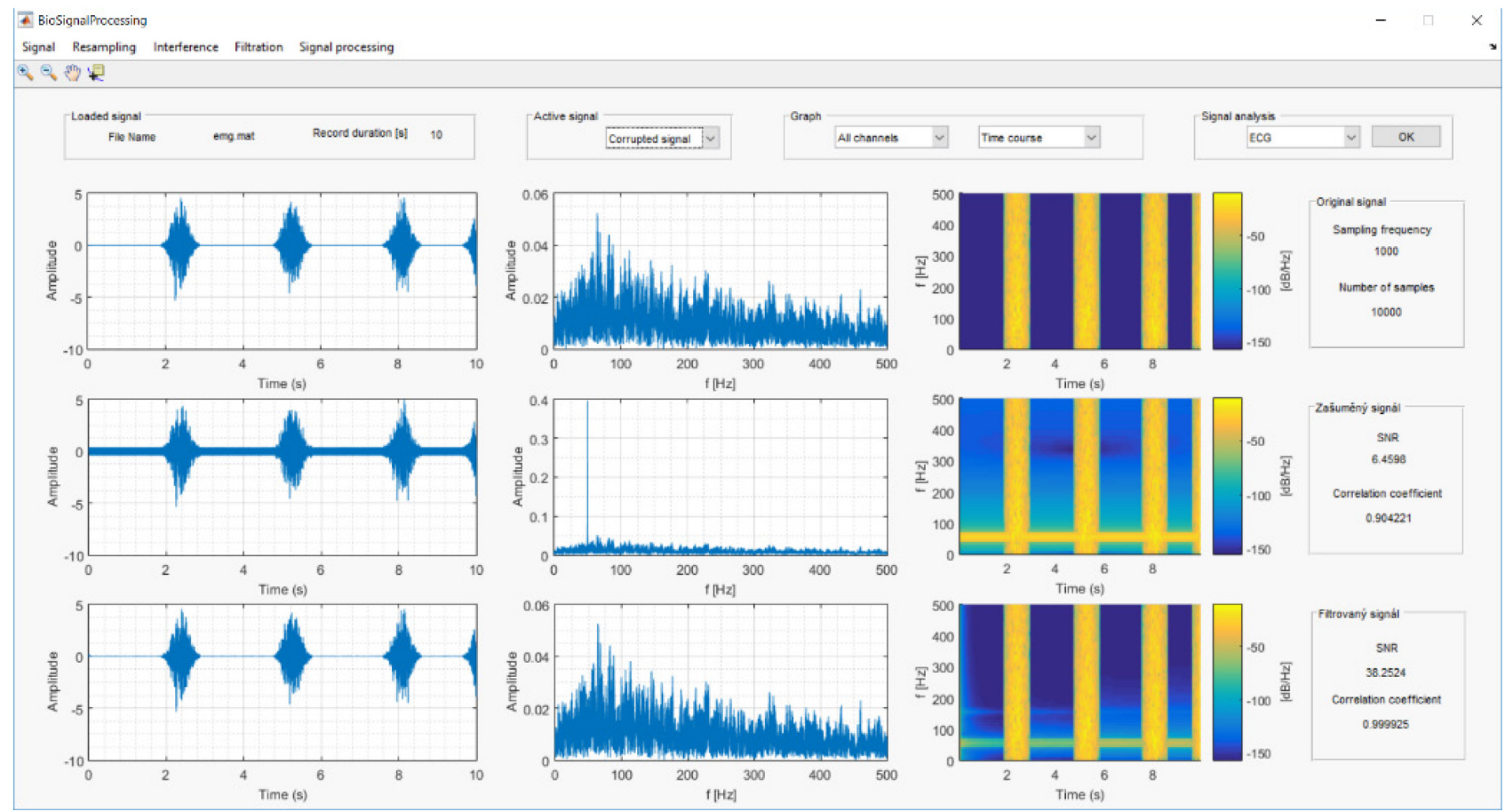

Fig. 1. MATLAB software GUI. Software enables corrupting of the signal with many types of the interferences and artefacts, its filtering and other processing. Software was implemented as the tool for testing methods of biological signal processing and their comparison.

\subsection{Adaptive noise canceller}

The method of ANC filtration exploits two input signals $[7,8,9]$. The primary input is the combination of the original signal and the noise, whose removal is the target of the method. The secondary input is the synthetic reference, which is in correlation with the noise. The filter modifies the reference so that the signal very similar to the noise is gained and the output signal is obtained as its difference with the input signal. 
The output is connected to the locked loop of the filter so that minimal output power and also a small power of the noise in output signal are obtained. For the fast optimization of the input signal weights, the RLS (Recursive Least Square) filter [14,15,16] which uses the sum of the squares of the error signal (deviation of the reference signal and the input signal) as a critical function, is used $[1,3]$.

\subsection{Wavelet transform}

Because of many limitations of the Fourier transform used in the processing of the non-stationary signals, the methods of the time-frequency processing are found. The idea of the WT is the suitable changing of the maternal wavelet's width in time and to gain the optimal ratio between time and frequency distinguishability $[10,11,12]$.

The WT technique is very often used in the processing of the non-stationarity signals but also in other cases, because of the extensive selection of the maternal waves, whose shape often responds to their main characters (e.g. QRS complex of ECG) [4,5]. According to [6], many types of maternal wavelets were tested and three of them were evaluated in the study (Db6, Sym4 and Meyer wavelet).

\section{Criteria of Performances}

The three methods discussed in the previous section are applied to the corrupted EMG signal. The criteria used for the performance analysis of each method are SNR and shape distortion of the output signal.

\subsection{Signal generation}

The simulated EMG signal was generated using LabView Biomedical toolkit [18] and has the following properties:

- maximum amplitude $4 \mathrm{mV}$,

- contraction duration $1 \mathrm{~s}$,

- frequency bandwidth $20-300 \mathrm{~Hz}$,

- sampling frequency $1 \mathrm{kHz}$.

First, the $50 \mathrm{~Hz}$ PLI with amplitude $0.4 \mathrm{mV}$ is added to the generated EMG signal. This signal has the SNR value of $6.459 \mathrm{~dB}$. Then, the corrupted EMG signal is filtered using the methods discussed above, and SNR and correlation coefficient with the original signal are calculated. For the graphic representation, also the Bland-Altman analysis is shown for each method.

\subsection{Signal-to-noise ratio}

The value of SNR is defined as the ratio of the simulated signal power $\left(\mathrm{Ps}_{\mathrm{S}}\right)$ to the power of the remaining interference $\left(\mathrm{P}_{\mathrm{N}}\right)$ according to $(2)$.

$$
S N R=10 \cdot \log \frac{P_{S}}{P_{N}}
$$

The SNR value is calculated for the signal after adding the interference and then, for each filtering method to compare, how much the method was successful. With the increasing SNR, the power of the noise is smaller, and the filtering process is more quality. 


\subsection{Correlation coefficient}

The correlation coefficient is also computed for the signal with interference and the pair of the output signal and the original simulated signal. The value of the correlation coefficient ranged from 0 to 1 shows the shape similarity of the filtered signal and the original signal. Higher correlation coefficient means a lower signal's shape distortion.

\subsection{Bland-Altman analysis}

Bland-Altman graph $[12,13]$ is used mainly to compare the results of all methods. The chart shows the relation between the result of the signal filtration and the original signal. Bland-Altman analysis compares two variables in their difference and aver-age, which are computed for each sample. The resulting graph is the dependence of this difference and average. The average value (AVG) and the standard deviation (SD) are computed and shown in the graph as three horizontal lines (AVG, AVG $\pm 1.96 \cdot \mathrm{SD})$.

More samples located around the AVG horizontal line shows the more accurate relation between the original and the output signal.

\section{Results}

Each of the three methods mentioned in section 2 gained different results that are presented in this section.

Notch filter. The classical IIR notch filter [17] eliminates the spectral lines of the selected frequency components but also reduces the power of the nearby frequencies. The sufficient attenuation of the PLI frequency can be obtained by increasing the width of the stopband. However, the wider stopband causes more information removal and the signal is distorted. In this study, the filter orders 1, 5 and 10 were used. In the case of the order 1, SNR descended beside the original value but using the higher order SNR increased. On the other hand, the correlation achieved quite high value in all cases.

Adaptive noise canceller. In the case of the ANC filtration method, SNR in-creased very highly, and the correlation was preserved. Because ANC works with the reference which in this case entirely copies the interference signal, the signal is not distorted in any way and only the PLI is removed. The order of the adaptive filter was set to 1,5 and 10. The highest SNR was gained in the case of the order 1, with increasing order SNR decreased.

Wavelet transform. From the available types of maternal waves in MATLAB software, the Db6, Sym4 and Meyer wave gained better result. However, SNR decreased to zero and correlation coefficient reached only about 0.24 . Though WT is a recommended method for the processing of the non-stationary signals, in this case this method reached the lowest results in SNR and correlation because of the elimination of the higher frequencies of the signal and the information loss. 
(a)



(c)

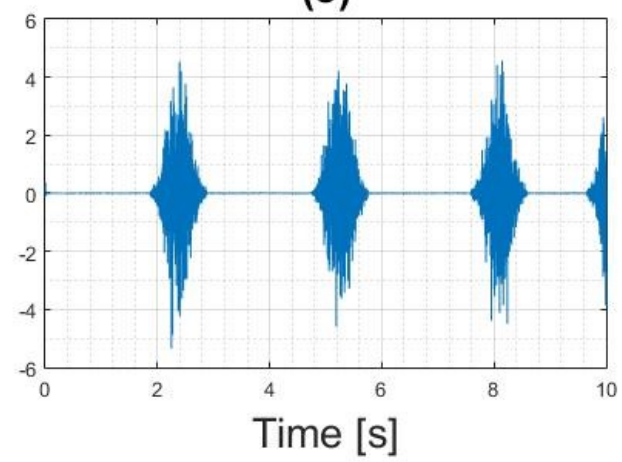

(b)

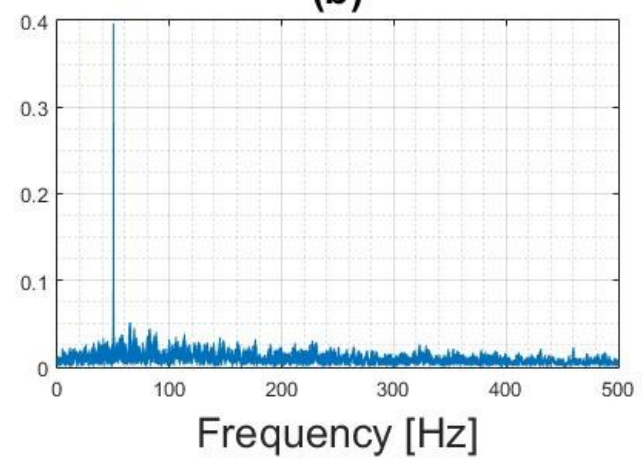

(d)

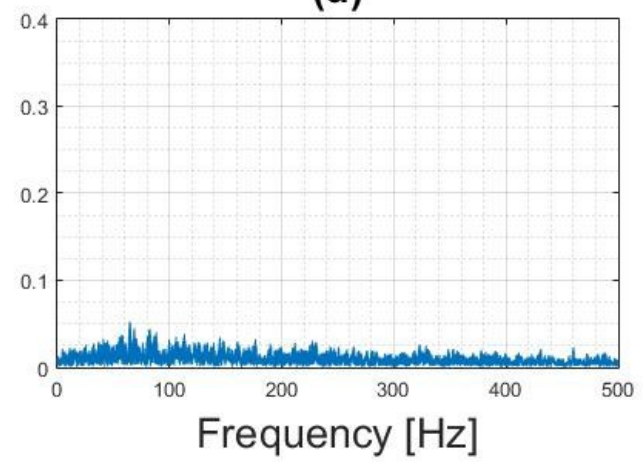

Fig. 2. Time waveforms and frequency spectra of the signal before $(a, b)$ and after $(c, d)$ filtering using ANC method.

Table 1. Performance results for each method.

\begin{tabular}{cccc}
\hline \multicolumn{2}{c}{ Method } & SNR [dB] & $\begin{array}{c}\text { Correlation } \\
\text { coefficient }\end{array}$ \\
\hline Notch & 1 & 5.847 & 0.8601 \\
& 5 & 12.11 & 0.9687 \\
& 10 & 15.33 & 0.9852 \\
ANC & 1 & 38.25 & 0.9999 \\
& 5 & 35.78 & 0.9999 \\
& 10 & 36.36 & 0.9999 \\
WT & Db6 & 0.2617 & 0.2418 \\
& Sym4 & 0.2457 & 0.2345 \\
& Meyer & 0.2437 & 0.2336 \\
\hline
\end{tabular}



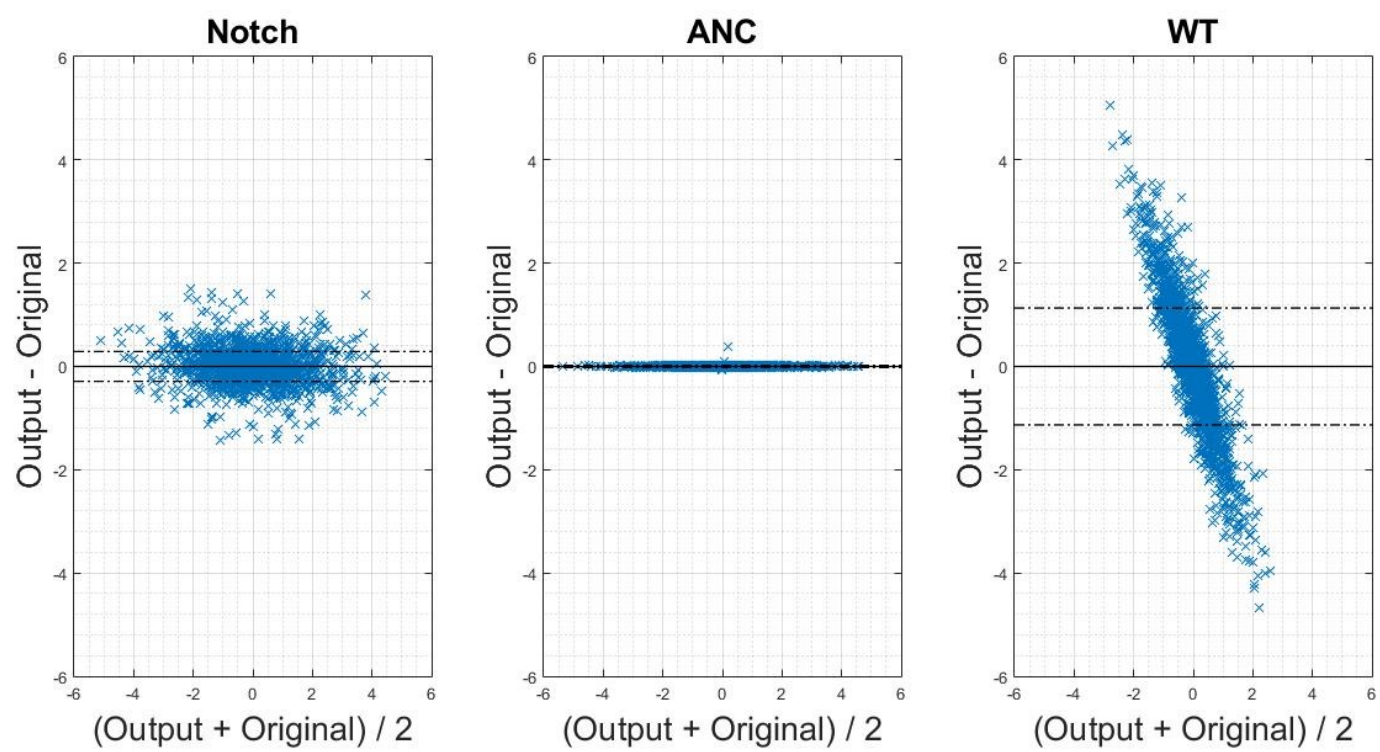

Fig. 3. Bland-Altman graphic analysis of each method shows its accordance with the original EMG signal. The graph of the ANC method indicates the best result because of the lowest difference values in all samples of the signal.

The results indicate, that from the selection of methods used in this study, the ANC filtration method is applicable in accordance to eliminate PLI from EMG signal. It gained the best results and the correlation is still close to 1 , so it has the minimum effect on the shape of the signal.

\section{Conclusion}

In the study, three methods used for reducing PLI in the EMG signal are compared. For the analysis of their performance, SNR and correlation coefficients are computed as indexes of each technique. The SNR values of the signal containing PLI and the output signal show the accuracy of each method. Concerning SNR and correlation coefficients, ANC filter proves to be the most suitable method of EMG signal processing in the case of PLI removal.

\section{Acknowledgment}

This article was supported by the Ministry of Education of the Czech Republic (Project No. SP2018/170). This work was supported by the European Regional Development Fund in the Research Centre of Advanced Mechatronic Systems project, project number CZ.02.1.01/0.0/0.0/16_019/0000867 within the Operational Programme Research, Development and Education.

\section{References}

[1] R. M. Rangayyan, Biomedical signal analysis, New Jersey: John Wiley \& Sons. 33 (2015).

[2] E.N. Bruce, Biomedical signal processing and signal modeling, New York: Wiley. (2001) 335336.

[3] S.D. Soedirdjo, K. Ullah, R. Merletti, Power line interference attenuation in multi-channel sEMG signals: Algorithms and analysis, In: EMBC (2015) 3823-3826.

[4] M. Reaz, M.S. Hussain, F. Mohd-Yasin, Techniques of EMG signal analysis: detection, processing, classification and applications, Biological Procedures Online. 8 (2006) 11-35.

[5] P.S. Addison, The illustrated wavelet transform handbook: introductory theory and applica-tions in science, engineering, medicine and finance, CRC press (2017). 
[6] M. Hussain, M. Reaz, F. Mohd-Yasin, M. Ibrahimy, Electromyography signal analysis us-ing wavelet transform and higher order statistics to determine muscle contraction, Expert Systems. 26 (2009) 35-48.

[7] R. Martinek, J. Konecny, P. Koudelka, J. Zidek, H. Nazeran, Adaptive optimization of con-trol parameters for feed-forward software defined equalization, Wireless Personal Commu-nications. 95 (2017) 4001-4011.

[8] R. Martinek, M. Kelnar, J. Vanus, P. Koudelka, P. Bilik, J. Koziorek, J. Zidek, Adaptive noise suppression in voice communication using a neuro-fuzzy inference system, In: Tele-communications and Signal Processing. (2015) 382-386.

[9] A. Sugiyama, Adaptive noise canceller with two SNR estimates for stepsize control, In: Consumer Electronics. (2018) 1-2.

[10] R. Martinek, R. Kahankova, J. Nedoma, M. Fajkus, K. Cholevova, Fetal ECG Prepro-cessing Using Wavelet Transform, In: Proceedings of the 10th International Conference on Computer Modeling and Simulation. (2018) 39-43.

[11] P.S. Addison, The illustrated wavelet transform handbook: introductory theory and applica-tions in science, engineering, medicine and finance, CRC press (2017).

[12] R. Martinek, R. Kahankova, J. Jezewski, R. Jaros, J. Mohylova, M. Fajkus, M., J. Nedoma, H. Nazeran, Comparative Effectiveness of ICA and PCA in Extraction of Fetal ECG From Abdominal Signals: Toward Non-invasive Fetal Monitoring. Frontiers in physiology. 9 (2018).

[13] D. Giavarina, Understanding bland altman analysis. Biochemia medica: Biochemia medica. (2015) 141-151.

[14] R. Martinek, J. Zidek, P. Bilik, J. Manas, J. Koziorek, H. Wen, The use of lms and rls adaptive algorithms for an adaptive control method of active power filter, Energy and Power Engineering. 5 (2013) 1126-1133.

[15] R. Kahankova, R. Martinek, P. Bilik, Non-invasive Fetal ECG Extraction from Maternal Abdominal ECG Using LMS and RLS Adaptive Algorithms, In: International Afro-European Conference for Industrial Advancement. (2016) 258-271.

[16] R. Kahankova, R. Martinek, P. Bilik, Fetal ECG extraction from ab-dominal ECG using RLS based adaptive algorithms, In: Carpathian Control Conference. (2017) 337-342.

[17] H.K. Jayant, K. Rana, V. Kumar, S. Nair, P. Mishra, Efficient IIR notch filter design using Minimax optimization for $50 \mathrm{~Hz}$ noise suppression in ECG, In: Signal Processing, Computing and Control. (2015) 290-295.

[18] A. Deshmukh, Y. Gandole, ECG feature extraction using NI LAB-VIEW biomedical workbench, International Journal of Recent Scientific Research, 6 (2015) 5603-5607. 